\title{
Anatomy Transfer
}

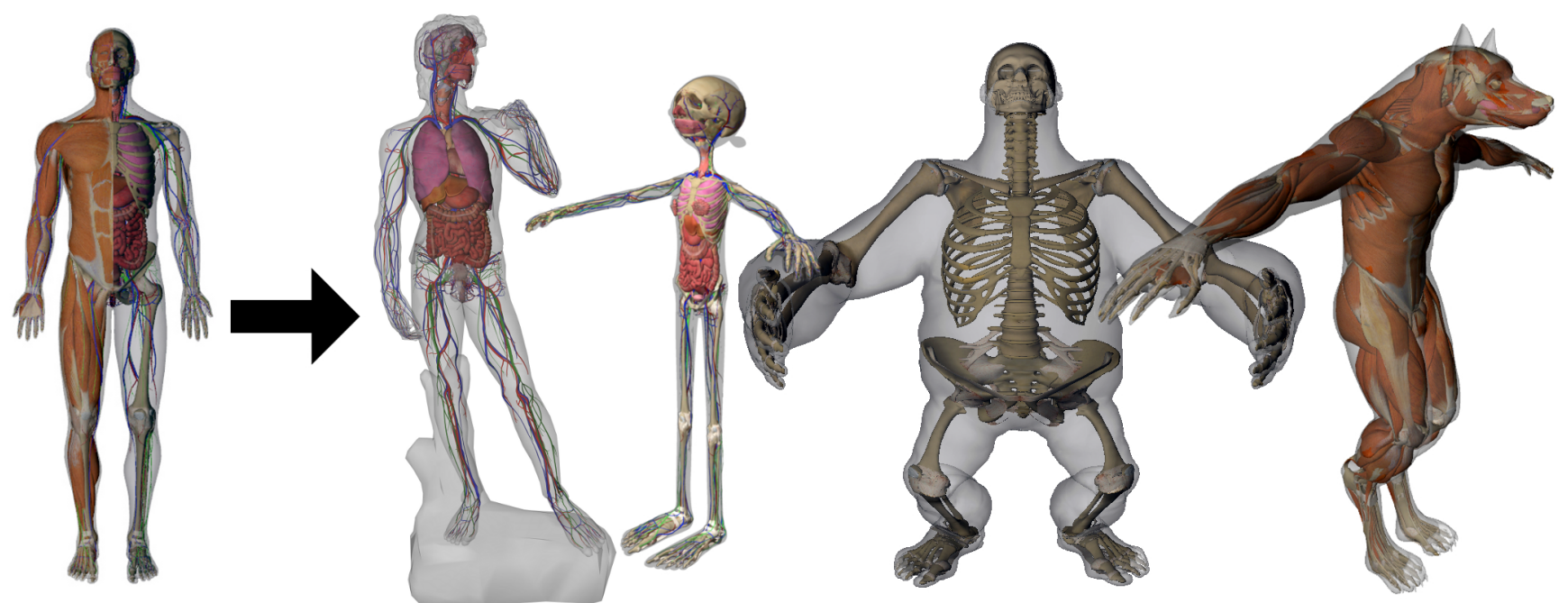

Figure 1: A reference anatomy (left) is automatically transferred to arbitrary humanoid characters. This is achieved by combining interpolated skin correspondences with anatomical rules.

\begin{abstract}
Characters with precise internal anatomy are important in film and visual effects, as well as in medical applications. We propose the first semi-automatic method for creating anatomical structures, such as bones, muscles, viscera and fat tissues. This is done by transferring a reference anatomical model from an input template to an arbitrary target character, only defined by its boundary representation (skin). The fat distribution of the target character needs to be specified. We can either infer this information from MRI data, or allow the users to express their creative intent through a new editing tool. The rest of our method runs automatically: it first transfers the bones to the target character, while maintaining their structure as much as possible. The bone layer, along with the target skin eroded using the fat thickness information, are then used to define a volume where we map the internal anatomy of the source model using harmonic (Laplacian) deformation. This way, we are able to quickly generate anatomical models for a large range of target characters, while maintaining anatomical constraints.
\end{abstract}

CR Categories: I.3.7 [Computer Graphics]: Three-Dimensional Graphics and Realism-Animation

Keywords: Character modeling

\section{Introduction}

A high level of anatomical precision is necessary in many Computer Graphics applications, from visualizing the internal anatomy for education purposes, to anatomical simulation for feature films, ergonomics, medical, or biomechanical applications (e.g. optimizing muscle energy). Highly realistic animations showing muscles or tendons deforming the skin typically require precise anatomical models. Moreover, the control of the fat distribution is important for achieving the associated secondary dynamics effects. While a lot of research addresses the challenge of fast and accurate simulation, we focus on the upstream part of the pipeline, modeling anatomy.

The current tools available for artists to model anatomical deformations [Maya-Muscle 2013] as well as early academic work [Wil- helms and Van Gelder 1997; Scheepers et al. 1997] extensively rely on user input, essentially amounting to setting up the musculature from scratch. Recent years witnessed huge improvements in anatomically-based simulation, especially in terms of computational efficiency [Patterson et al. 2012]. However, the cost of setting up a $3 \mathrm{D}$ anatomical model for a given character remains. This task is very time consuming and tedious, as it requires modeling of the bones, organs, muscles, and connective and fat tissues. With real humans, it is possible to take advantage of $3 \mathrm{D}$ imaging, such as MRI [Blemker et al. 2007]. However, this route is difficult or even impossible for fictional characters, ranging from Popeye to Avatar's Na'vi.

A naive idea to solve the problem would be to transfer the anatomy from a reference character to the target in a purely geometric way. It is obvious this route has a number of shortcomings: humanoids are made of bones, viscera, muscles, and fat tissues. Specific anatomical rules need to be preserved in order to generate a plausible anatomical structure: bones should remain straight and symmetric, and the distribution of fat, which may vary from one individual to another, should be taken into account while transferring muscles and viscera. CG characters can also contain non-anatomical or stylized components, such as hair, a shell, or even clothes. A specific problem is to prevent the internal anatomical structure to fill these areas, as we want our method to work even in these challenging cases.

We propose a semi-automatic method for creating the internal anatomy of any target character by transferring the internal anatomy of a highly-detailed anatomical model with minimal fat layers (Zygote body). Our method starts by registering the skins (outer boundaries) of the two models. An initial deformation between the two volumes is established using Laplacian deformation. The Laplacian is however uninformed about the anatomy and can, e.g., bend or otherwise unnaturally deform the bones. Therefore, we impose a number of anatomical constraints, such as requiring the bones to remain quasi-rigid. We also provide a tool for carving out the fat layers as well as the non-anatomical parts of the volume of the target model, before transferring the muscles and viscera. Our specific contributions are:

- a novel registration method to transfer a source anatomy to 
characters with very different shapes while exploiting anatom- 135 ical knowledge to get a plausible result;

- the use of a texture, specifying non-uniform distribution of fat under the skin of a character, and a robust method to erode the internal volume accordingly;

- a user-friendly tool for editing the fat distribution texture, if 139 needed, on a per bone basis.

We exploit prior knowledge about human anatomy, e.g., we require ${ }_{140}$ that bone shapes and sizes remain as close as possible to human, by restricting the deformation modes and enforcing symmetry during ${ }_{142}$ registration.

The journey towards realistic Computer Graphics humans starts with modeling. To our knowledge, this work is the first attempt to address the challenging goal of semi-automatic anatomy authoring. While many limitations and open questions remain, we hope that our method opens the door to inexpensive anatomy authoring tools and helps to promote and democratize applications leveraging anatomically-based simulation and visualization.

\section{Related work}

Skeleton-based models have been used in computer graphics to control the motion of the human body or its interaction with objects using joint torques, see e.g. [Faloutsos et al. 2001; Zordan et al. 2005] for full body, and [Pollard and Zordan 2005; Kry and Pai 2006] for the hand. [Baran and Popović 2007] presented a method for automatic rigging of character skins without internal anatomy, except for automatically inferred animation skeleton. Musculoskeletal models have been proposed to animate muscle deformations [Wilhelms and Van Gelder 1997; Scheepers et al. 1997; Aubel and Thalmann 2001], to perform facial animation [Waters 1987; Sifakis et al. 2005], to study or improve the control [Lee and Terzopoulos 2006; Wei et al. 2010; Wang et al. 2012], or to increase the quality of the flesh and skin deformations [Lee et al. 2009]. Beyond bones and muscles, [Sueda et al. 2008] demonstrated an impressive model including detailed bones, joints, skin, and tendons. The deformations of the skin due to the tendon actuators dramati- 167 cally improve the resulting quality. The windpipe is visible in an 168 increasing number of feature animation characters, and the veins increase the realism of the skin.

While encouraging results have been demonstrated for transferring deformations from one model to another [Sumner and Popović 2004], little has been done in terms of volumetric geometry transfer across shapes. A lot of effort has been dedicated to solving the registration problem: the computation of correspondences between objects, mainly between surface meshes or images. Registration is a fundamental problem in computer science, especially in computer graphics [Kaick et al. 2011]. Most registration methods alternate between two steps: A) the estimation of sparse correspondences, optimizing the extrinsic (e.g., closest points [Besl and McKay 1992]) or intrinsic (e.g., [Bronstein et al. 2008]) similarity; and B) correspondence completion and regularization to achieve plausible dense displacement fields. To improve the robustness of the registration with respect to object poses (i.e., rigid transforms, isometry, etc.), different isometry invariant parameterizations have been proposed such as spectral embedding [Mateus et al. 2008], conformal mapping [Lipman and Funkhouser 2009], or functional maps [Ovsjanikov et al. 2012]. On the other hand, robustness to topological noise and to partial data can be achieved ${ }_{187}$ from extrinsic correspondences (i.e. established in Cartesian space) 188 [Li et al. 2008; Huang et al. 2008]. For regularization, a displace- 189 ment model is often associated: ranging from rigid, affine, to as- 190 rigid-as-possible deformation fields, possibly with extra constraints 191 such as articulations [Gilles et al. 2010]. Our method can be seen as a partial registration process, where skin surfaces are first registered based on the data, and the interior estimated using interpolation and anatomical rules.

\section{Overview}

The anatomy of a living body depends on numerous physiological constraints. The huge variability of anatomy is constrained by critical anatomical rules. We propose semi-automatic modeling of humanoid anatomy that uses these rules to constrain the resulting volumetric deformation, aiming to achieve as-anatomical-as-possible results. For the skeleton, our pipeline relies on the rule that bones must stay straight at the end of the anatomy transfer (R1), and symmetric across the sagittal plane (R2). The third rule is the fact that there is no relation between the quantity of fat tissue and the size of the bones [Moore and Dalley 1999]. For example, a fat character has the same skeleton as a lean one (R3), but the muscularity is proportional to keep up the body (R4) [M. Gilroy 2008]. The fat tissues are localized mainly between the skin and the muscles (R5) [M. Gilroy 2008]. They can be interpreted as a stock of energy and therefore, the amount of fat tissue can be very variable. During anatomy transfer, anatomical structures cannot disappear (R6), and the muscular insertion points are preserved (R7).

Our anatomy transfer pipeline implementing these rules is illustrated using a didactic anatomy piece in Fig. 2. The user provides the skin of a target character and may add a user-defined distribution of sub-skin fat (possibly including other non-anatomical structures) modeled using a thickness function in texture space (Fig. 2.a). As an alternative to user defined fat map, this information can be also extracted from real MRI data. Our method requires that the source (reference character) and target skin share the same $(u, v)$ texture space. The first step, not shown in the figure, is thus to compute the registration of the source and the target skin (Sec.4).

Our source model (Fig. 2.c) is composed of bones, skin, muscles and viscera, and it includes almost no fat. We therefore erode the volume of the target (Fig. 2.b) according to the thickness of the fat layer, to warp our "lean" source anatomy to the sub-fat part of the target volume (Sec. 5), following rule (R5). The user can create the thickness data for stylized and cartoony characters using our new semi-automatic tool (Sec. 6).

The displacement of the skin from the source to the eroded target is then interpolated within the volume to transfer the internal anatomy (Sec. 7). This, along with a reasonable choice of fat thickness, enables us to follow rules (R3) and (R4). However, naively interpolating the skin deformation generally results in visible artifacts in the internal anatomy, especially in the skeleton, which may exhibit bent or inflated bones. We thus use this interpolation (Fig. 2.d) as an attractor for a constrained registration (Fig. 2.f), where the constraints express anatomical properties, such as the symmetry of the skeleton about the sagittal plane (Sec. 8). This allows us to incorporate rules (R1) and (R2). This constrained registration provides us with a plausible skeleton which fits the shape of the target character while following the anatomical rules.

Finally, we compute a new interpolating deformation field, using the internal skeleton as well as the eroded shape as boundary conditions (Fig. 2.e). This allows us to interpolate the remaining anatomical entities in between. This preserves all the anatomical structures and their relative locations, and satisfies rules (R6) and (R7). 


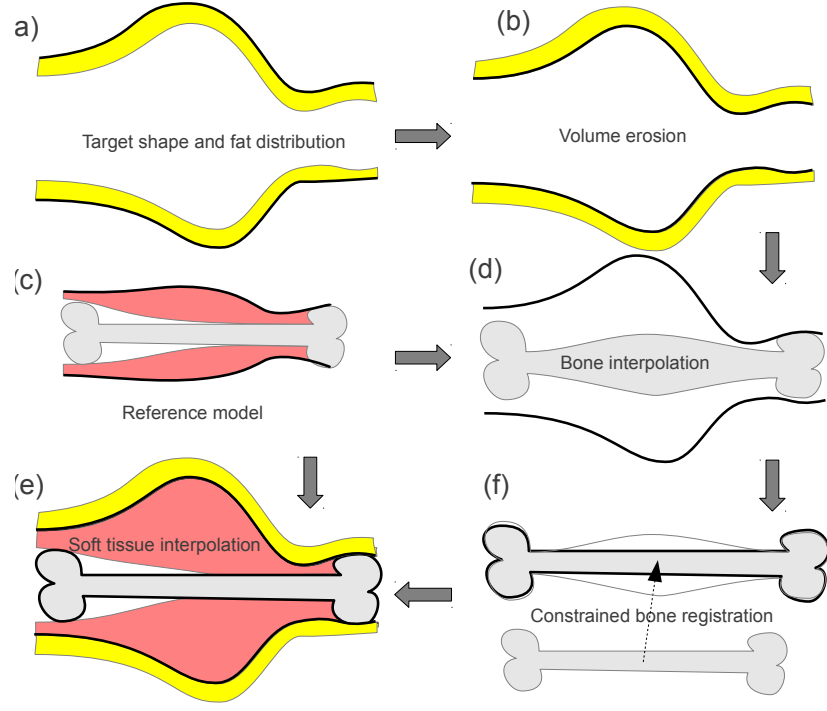

Figure 2: Anatomy transfer pipeline.

\footnotetext{
${ }^{1}$ www.zygote.com
}

The first step of our pipeline is to establish surface correspondences between the source and target skins. Because skins of different subjects are not isometric, we focus on extrinsic correspondences for registration. For simplicity, we compute closest point correspondences such as in the popular Iterative Closest Point algorithm [Besl and McKay 1992]. Based on correspondences established at each iteration, a smooth as-rigid-as-possible deformation field for the source skin is updated. As in [Gilles et al. 2010], we use the shape matching deformation method [Müller et al. 2005] which is both efficient (being based only on geometry) and controllable. Skin stiffness is progressively decreased during the registration to reduce sensitivity to local minima. Manual initialization is performed in the case of large differences between the pose of the source and target characters.

\section{Volume erosion}

The internal volume of the target character is composed of the skeleton and the soft tissues modeled in the source anatomy, along 269 with a significant volume of fat tissue, which is usually not explic- 270 itly represented in anatomical models, including ours ${ }^{1}$, and therefore difficult to model. We thus consider only a sub-skin layer of the fat tissue, which separates the skin from the rest of the anatomy. This layer, which may have a significant thickness depending on the target character, reduces the available volume for the skeleton and muscles. The layer of fat below the skin is not uniform around the body. It is well-known that men and women exhibit different distributions, and this distribution may also vary between individuals [Gray and Lewis 1918]. With realistic human models, we make the simplifying assumption that each gender can be associated with one scalable distribution.

The simplest way to model the distribution of fat is to add a channel to the texture of the skin to represent the local thickness of the fat layer. We compute this thickness using the MRI image of a real person. We first tag the voxels corresponding to the skin and to the fat layer using a segmentation technique. Relying on the local normal to associate each voxel of the skin to a thickness would not be reliable due to skin curvature and imperfections in the input data. We therefore rely on discrete data, exploiting voxel neighborhoods. We compute a forest of shortest paths from the skin voxels to the fat voxels, where each skin voxel is the root of a tree. Then for each skin voxel we set the local fat thickness to the maximum distance to the leaves of its tree. Based on the texture coordinates of the skin voxels and the associated thickness, we interpolate the value at each pixel of the thickness texture.

The thickness texture can be edited as discussed in Section 6, and then used to perform volume erosion (Fig. 2.b). For each vertex of the target, we compute the local depth using the texture coordinates and we move the vertex by this distance following the forest of shortest paths. An example result is shown in Fig. 5.

\section{Edition of the fat distribution texture}

In order to generate fat distribution textures for arbitrary characters, we created a "fat editor" that provides both physically plausible initialization and full artistic control. Based on the observation that fat distribution is close to uniform around each bone, we adopted the idea of bounded biharmonic weights [Jacobson et al. 2011] to create smooth fat distribution maps. We set the bones as boundary constraints and minimize biharmonic (Laplacian) energy subject to these constraints. This way, we smoothly spread influence from the bones to the skin and obtain a fat map by tuning only a few parameters. Choosing a reasonable thickness leaves space for a realistic amount of muscle tissue (rule R4).

Fig. 3 shows the pipeline of our fat editor. The editor first loads the target character model and its bones calculated using our bone registration. Similarly to [Kavan and Sorkine 2012], we compute bounded biharmonic weights using a regular voxel grid, obtained using the Binvox program [Binvox 2013]. After pre-computing the weights, users can very quickly tune the amount of fat distribution around each bone. For example, we can assign 0.4 to the pelvis and sacrum bones to model the fat around the character belly, but give 0 to the skull because there is no fat beneath his scalp. The editor computes linear combination of pre-computed bone weights with the control parameters set by the users to generate the final fat distribution map.

The fat editor does not have to use only the anatomical bones. If artists want to control the fat around a certain region with more details, they can add fictional bones inside that region and tune the new parameters introduced by the fictional bones. We did not do this in our examples because we were satisfied with the results using only anatomical bones.

\section{Interpolation}

In our framework, volumetric interpolation is required at two stages of the method: 1) to initialize bones inside the eroded skin, and 2) when soft organs are transferred, using both the eroded skin and the bones as boundary conditions. This section describes the interpolation method we use in both cases.

Given boundary conditions on the displacement field, we solve for the displacements in the interior by minimizing the harmonic energy, also known as Laplace interpolation [Press et al. 2002]. The principle is to compute as linear as possible interpolation by requiring zero value of the Laplacian of the displacement field at each unconstrained voxel. The boundary displacements $\bar{f}$ are incorporated as hard constraints:

$$
\begin{aligned}
\nabla^{2} f(x) & =0, x \text { inside } \\
f(x) & =\bar{f}, x \text { on the boundary }
\end{aligned}
$$




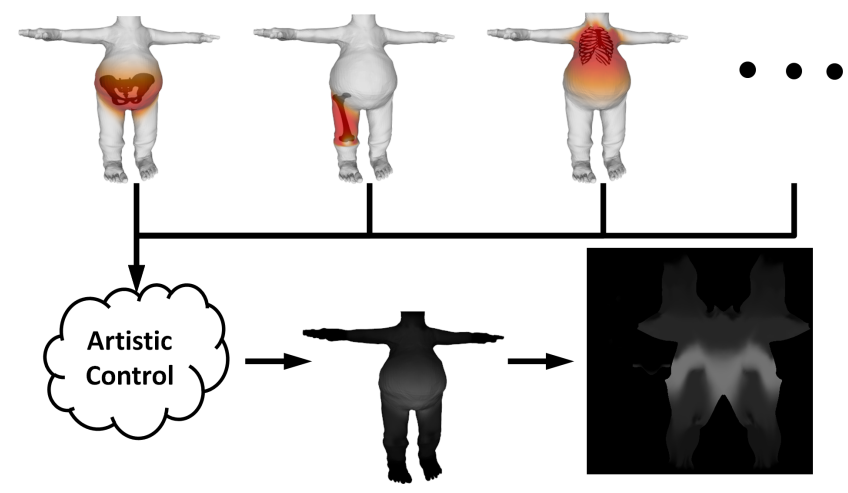

Figure 3: Fat distribution texture generation for the character. Top (initialization): we use bounded biharmonic weights to compute skin weights corresponding to each bone. Bottom (fat editing): artists can set fat parameters and generate fat distribution map. Brighter regions correspond to thicker fat layers.

The discretization on our grid results in a large sparse system of linear equations, which we solve using the Conjugate Gradient solver ${ }_{323}$ from the Eigen library [Guennebaud et al. 2010] . More sophisticated methods such as a multigrid solver with an efficient handling 324 of irregular boundaries [Zhu et al. 2010] could be used to further ${ }_{325}$ accelerate the computation.

\section{Bone registration}

Bones directly deformed using the method presented in Section 7 may become non-realistically stretched or bent, as illustrated in Fig. 2.d . The difference of shape between real or plausible characters and the reference anatomy is due to a different size as well as a different amount of soft tissue around them. Changes of character size mainly scales up or down the bones, while the changes of soft tissue do not modify the bones. We thus restrict each bone transformation to an affine transformation, using the initial interpolated bone as an attractor to a plausible location inside the body. Moreover, the symmetry of the trunk is enforced by deforming it using transformations centered in the sagittal plane. We did not impose symmetry constraints for pairs of corresponding bones to allow the input of non-symmetric target characters, such as the David model in our examples. The constrained minimization is performed by attaching all the voxels of the reference bone to a common affine frame and attracting them to their interpolated position using linear springs. We have not noticed any visible artifacts due to the possible shearing modes introduced by the affine transformations. We use an implicit solver to ensure stability [Baraff and Witkin 1998]. Organ intersections do not occur when the interpolation is foldoverfree, which is the case in all our examples: during the semi-rigid bone registration, the offsets between the interpolated bones and the registered bones mostly occur in the off-axis directions, so we have not encountered any intersection. If necessary, this issue could be addressed using standard collision handling routines.

Fig. 4 illustrates the benefits of bone registration compared to simple interpolation. Notice the bent bones in the legs, the oddly in- 337 flated bones in the arms of the interpolated skeleton, as well as the 338 broken symmetry of the rib cage are fixed by the affine registration. 339 Moreover, the shape of the skull is influenced by the hair during 340 the interpolation. This deformation is also filtered out by the affine 341 transformation.

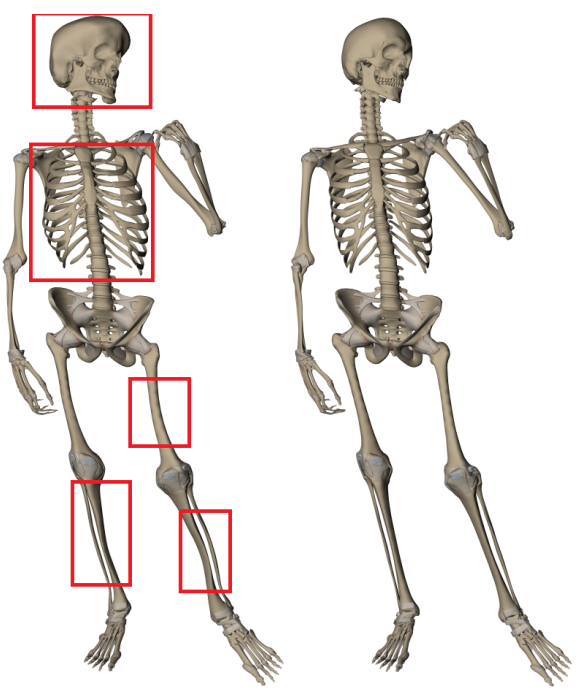

Figure 4: The benefits of bone registration. Left: after interpolation only. Right: after affine registration.

\section{Results}

We have successfully applied our framework to both realistic and cartoon characters, as can be seen in Fig. 1. Cartoon characters were not intended as a primary motivation for anatomically-based modeling, but they are a challenging stress test for the system, showing how far from the input model we can go.

A nice feature of our method is that what we actually compute a deformation field, which can be used to transfer arbitrarily complex internal geometry. Once this computation is achieved, we are able to transfer a complete anatomy including bones, muscles, ligaments, viscera, blood vessels, nerves etc. very quickly. Our fat editor allows an artist to tailor a distribution for a specific target character, as shown in Fig. 5. Other examples of anatomy transfer

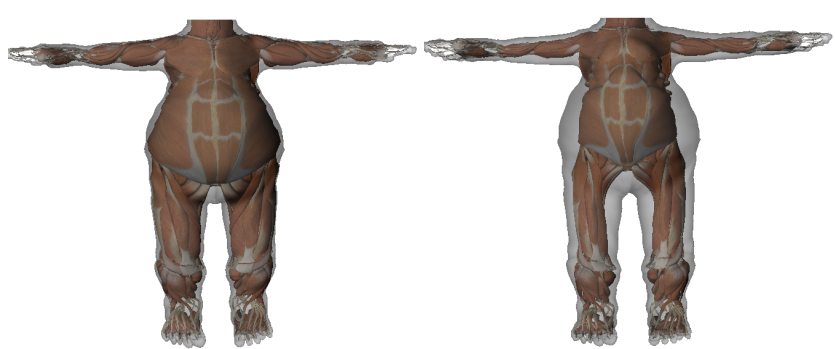

Figure 5: Transfer to a fat character. Left: without erosion. Right: a preliminary erosion accounts for the fat and results in a more plausible muscular system.

are shown in Fig. 6.

The reconstruction of Popeye in Fig. 7 exhibits a surprising chin, which could be mitigated using fat. Note, however, that his forearm bones are realistic despite the odd external shape. Fig. 7 also shows the reconstruction of the anatomy of Olive, a very thin character. We can notice how close her muscles are to her skin while her skeleton remains thin, but well adapted to her morphology. 

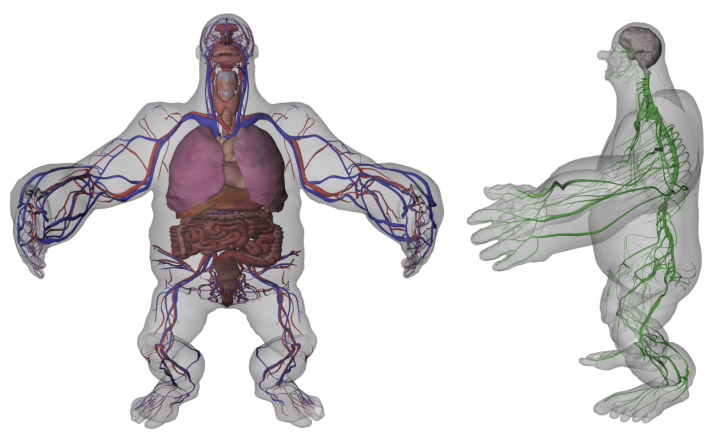

Figure 6: Brutus blood vessels and nerves.

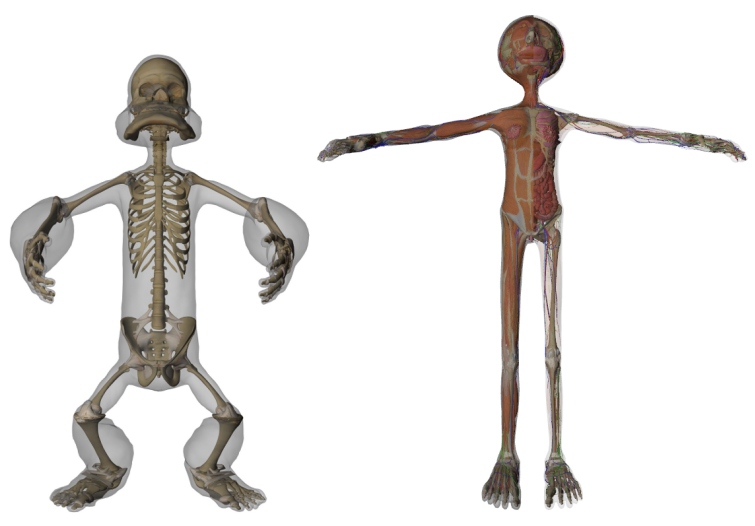

Figure 7: Popeye and Olive.

To see how far we can push the concept of anatomy transfer, we transferred our reference model into a werewolf (half human and half animal). Fig. 8 demonstrates how the human anatomy fits accurately within the body of this monster despite of the difference in morphology. The bottom of Fig. 8 validates the transfer by comparing the results we get with the musculoskeletal system of a real wolf (Canis lupus), shown on the left.

Fig. 9 shows the reconstruction of a real male based on his MRI image. The muscles are suprisingly well captured in the lower legs, the bottom cheeks and the trunk. Some muscles are not accurately reconstructed, due to different relative sizes in the real person and our reference model and to errors in skin registration. The latter are also responsible for inaccuracies in the fat layer. The goal of this reconstruction attempt is not to compete with established segmentation methods, but to suggest that anatomy transfer may provide a useful initial estimate. Moreover, a lot of thin anatomical structures which cannot be seen in the volumetric image are present in our model. In future work, complementing our framework with sparser but more accurate segmentation methods may provide useful constraints to insert in our interpolation, to accurately infer the positions of the features invisible in the MRI.

Our methods provides significant improvements over a shape matching method like [Gilles et al. 2010], which is based on different premises. They assume noisy MRI input and therefore employ approximate volumetric shape matching, while our method assumes exact correspondence between the input and the target surfaces, i.e., the deformation field has to interpolate rather than approximate the boundary. To make [Gilles et al. 2010] as interpolant as possible, we need to make the shape matching stiffness and clus- 410 ter size small enough, thereby slowing down the convergence and 411 requiring a sufficiently dense mesh. A comparison is shown in 412

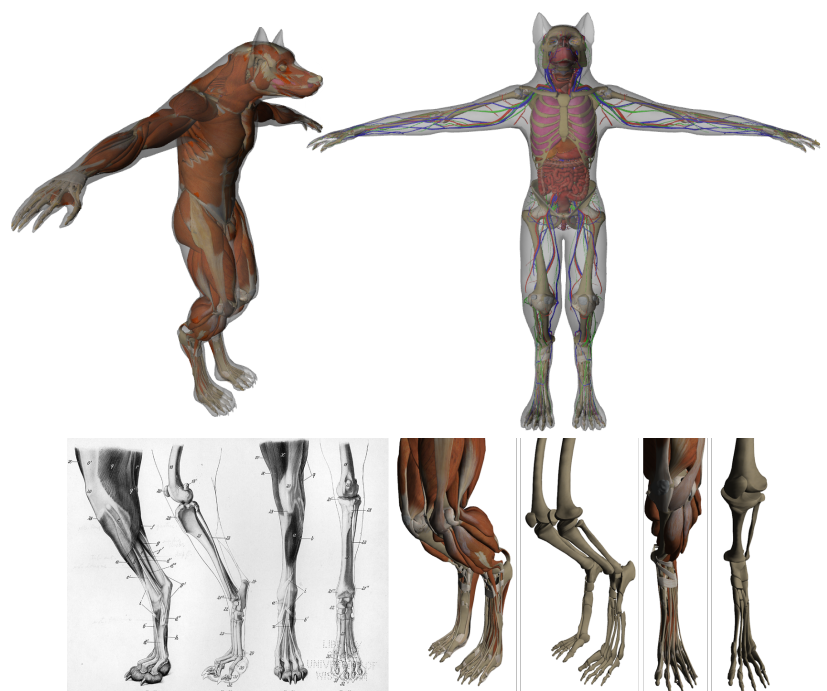

Figure 8: Top: Werewolf muscularity, skeleton, and internal organs. Bottom: Comparison between werewolf lower limb and real wolf lower limb.

method has a number of limitations. Firstly, automatically inferring non-standard distributions of fat from the morphology of the character would be an interesting extension. Standard human 

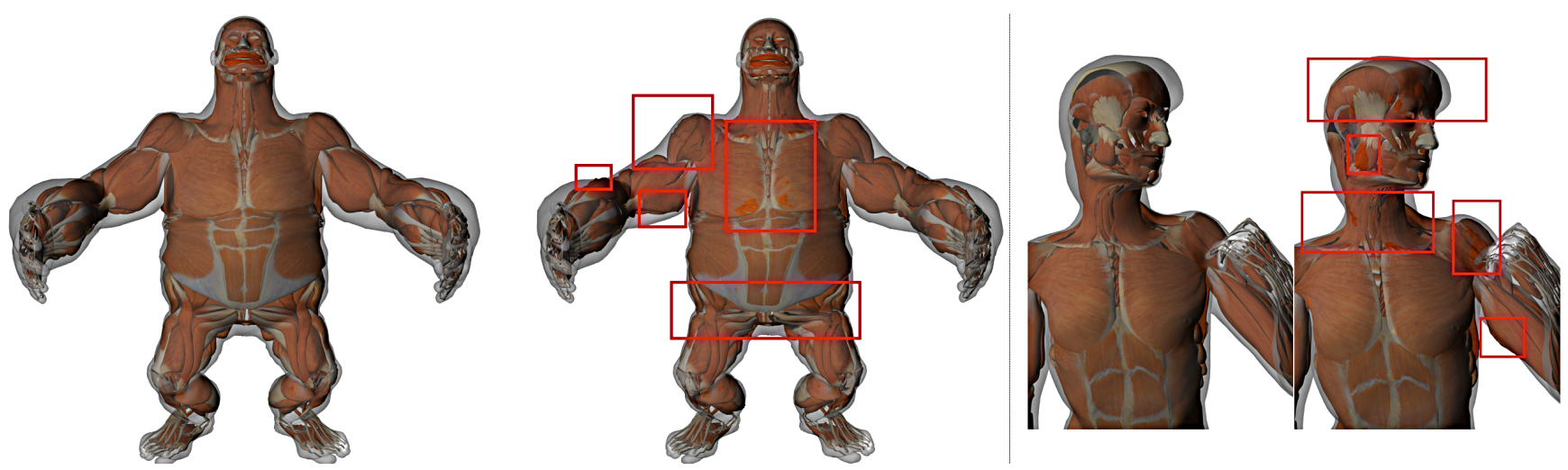

Figure 10: Left: our method. Right: the method of [Gilles et al. 2010] based on shape matching. Notice the artifacts of the latter method, e.g., the upper arm muscles intersecting the skin and asymmetry of the abdominal muscles.

morphograms (i.e. classes of shapes: big belly, big chest, or com- 45 pletely skinny) are available in the literature, but so far we found no precise information on the corresponding fat distribution. Moreover, we do not model the fat tissue distributed anywhere else than directly below the skin.

Other practical limitations are related to the registration. The skin correspondence is inferred on a proximity basis. This sometimes creates wrong results when the source and target characters are in different poses. Our volumetric interpolation method does not guarantee foldover-free displacement field: although we did not observe overlapping between internal structures in any of our examples, it could occur in theory. The skin registration fails when the target character has a different topology from the reference anatomy. For 462 the example shown in Fig. 5, we had to create a five-fingered variant ${ }_{463}$ of the target character.

\section{Conclusion}

To address the high costs associated with anatomy authoring, we have presented the first method for quickly creating a plausible anatomy for any target character. For realistic humanoid models, we transfer both the internal anatomical structures from a reference model, as well as the fat thickness information extracted and retargeted from MRI data. Our method is thus purely automatic. For ${ }^{471}$ cartoony characters, we offer a user friendly editing tool enabling 472 the user to tune the fat tissues of the target character. Transferring 473 the internal bones, viscera and muscles is then automatic.

We have shown that direct Laplace interpolation, perhaps sufficient 475 to generate simple effects such as muscle bulging, leads to objec- 476 tionable artifacts when used to transfer the full anatomy. Our spe- ${ }_{477}$ cific pipeline ensures that basic anatomical rules are preserved. $\quad 478$

In future work, we would like to take advantage of more anatomi- 479 cal knowledge to constrain the interpolations. We believe that our 480 method could also help the processing of body scans by computing ${ }^{481}$ a first guess to the segmentation process, and complementing the ${ }^{482}$ final result with thin structures, invisible in the volumetric image, ${ }^{483}$ as shown by our validation example (Fig. 9).

\section{Acknowledgements}

Many thanks to Laura Paiardini and Armelle Bauer for 3D modeling and kind support. We would also like to thank the anonymous reviewers for their detailed comments and feedback. This work was 48 partly funded by the French ANR SoHusim, the ERC Expressive 490 and CNRS Semyo projects.

\section{References}

Aubel, A., And Thalmann, D. 2001. Interactive modeling of the human musculature. In In Proceedings of Computer Animation, 7-8.

BARAFF, D., AND WitKin, A. 1998. Large steps in cloth simulation. In Proceedings of the 25th annual conference on Computer graphics and interactive techniques, ACM, New York, NY, USA, SIGGRAPH '98, 43-54.

BARAN, I., AND Popović, J. 2007. Automatic rigging and animation of 3d characters. ACM Trans. Graph. 26, 3 (July).

BESL, P., AND MCKAY, N. 1992. A method for registration of 3-d shapes. IEEE Trans. PAMI 14, 2, 239-256.

BINVOX, 2013. http://www.cs.princeton.edu/ min/binvox/.

Blemker, S., Asakawa, D., Gold, G., AND Delp, S. 2007. Image-based musculoskeletal modeling: Applications, advances, and future opportunities. Journal of Magnetic Resonance Imaging 25, 2, 441-451.

Bronstein, A., Bronstein, M., And Kimmel, R. 2008. $\mathrm{Nu}$ merical Geometry of Non-Rigid Shapes, 1 ed. Springer Publishing Company, Incorporated.

Faloutsos, P., van de Panne, M., and Terzopoulos, D. 2001. Composable controllers for physics-based character animation. In Proceedings of the 28th annual conference on Computer graphics and interactive techniques, ACM, New York, NY, USA, SIGGRAPH '01, 251-260.

Gilles, B., Reveret, L., AND PAI, D. 2010. Creating and animating subject-specific anatomical models. Computer Graphics Forum (June), http://onlinelibrary.wiley.com/doi/10.1111/j.14678659.2010.01718.x/abstract.

Gray, H., AND LEWIS, W. H. 1918. Anatomy of the human body. Philadelphia: Lea and Febiger, http://www.biodiversitylibrary.org/bibliography/20311.

Guennebaud, G., Jасов, B., ET AL., 2010. Eigen v3. http://eigen.tuxfamily.org.

Huang, Q.-X., Adams, B., Wicke, M., And Guibas, L. J. 2008. Non-rigid registration under isometric deformations. In 

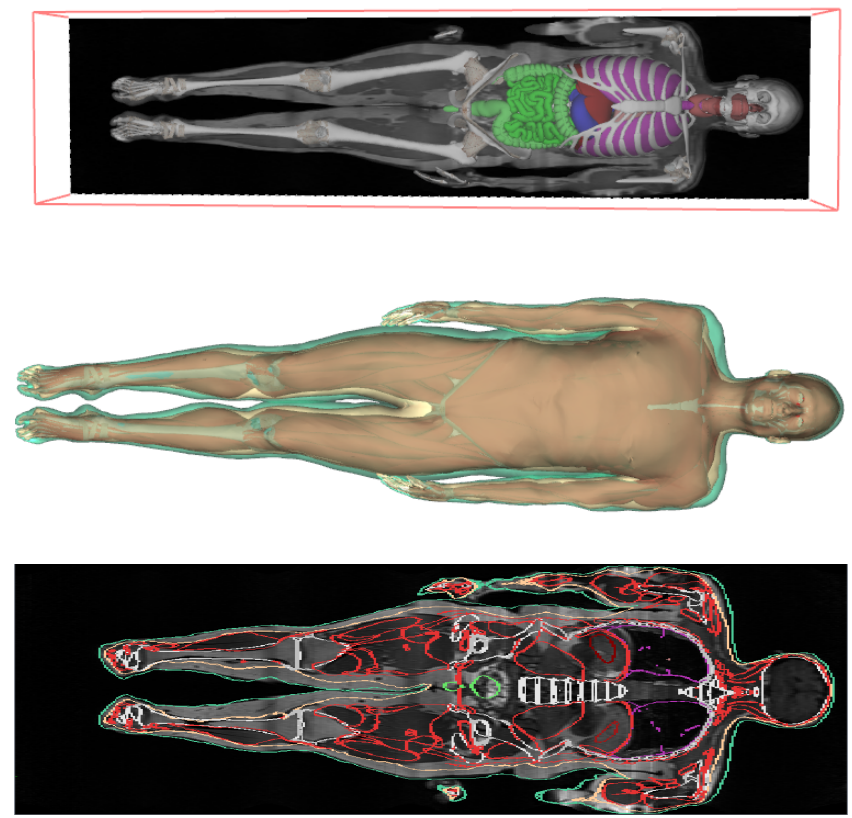

Figure 9: Transfer to an MRI image of a man laying on his back. Top: reconstruction of internal organs and skeleton within one slide of MRI Data. Center: reconstruction of muscular system. Bottom: comparison with the data. The green lines highlight our reconstructed surface, the beige lines correspond to the eroded volume, 517 while the red line is muscle reconstruction, white and gray lines are 518 bones and connectives tissues, the purple represents the lungs and the bright green represents the small intestine.

Proceedings of the Symposium on Geometry Processing, 1449- 522 1457.

Jacobson, A., BARAn, I., Popović, J., And Sorkine, O. ${ }^{524}$ 2011. Bounded biharmonic weights for real-time deformation. ${ }^{525}$ In ACM SIGGRAPH 2011 papers, ACM, New York, NY, USA, ${ }^{52}$ SIGGRAPH '11, 78:1-78:8.

Kaick, O. V., Zhang, H., Hamarneh, G., And Cohen-OR, D. 2011. A survey on shape correspondence. Computer Graphics Forum 30, 6, 1681-1707.

KAVAN, L., AND SORKINE, O. 2012. Elasticity-inspired deform- 531 ers for character articulation. ACM Transactions on Graphics (proceedings of ACM SIGGRAPH ASIA) 31, 6, 196:1-196:8.

Kavan, L., Collins, S., Zara, J., ANd O'Sullivan, C. 2008. 534 Geometric skinning with approximate dual quaternion blending. ACM Trans. Graph. 27, 4, 105.

KRY, P. G., AND PAI, D. K. 2006. Interaction capture and synthesis. ACM Trans. Graph. 25, 3, 872-880.

LeE, S.-H., AND Terzopoulos, D. 2006. Heads up!: biome- 539 chanical modeling and neuromuscular control of the neck. In 540 ACM SIGGRAPH 2006 Papers, ACM, New York, NY, USA, 541 SIGGRAPH '06, 1188-1198.

LeE, S., SifAKis, E., AND TERZOPOUlos, D. 2009. Comprehen- 5 sive biomechanical modeling and simulation of the upper body. 544 ACM Trans. Graph. 28 (September), 99:1-99:17.

Li, H., Sumner, R. W., AND PAUly, M. 2008. Global correspon- 546 dence optimization for non-rigid registration of depth scans. In ${ }_{547}$
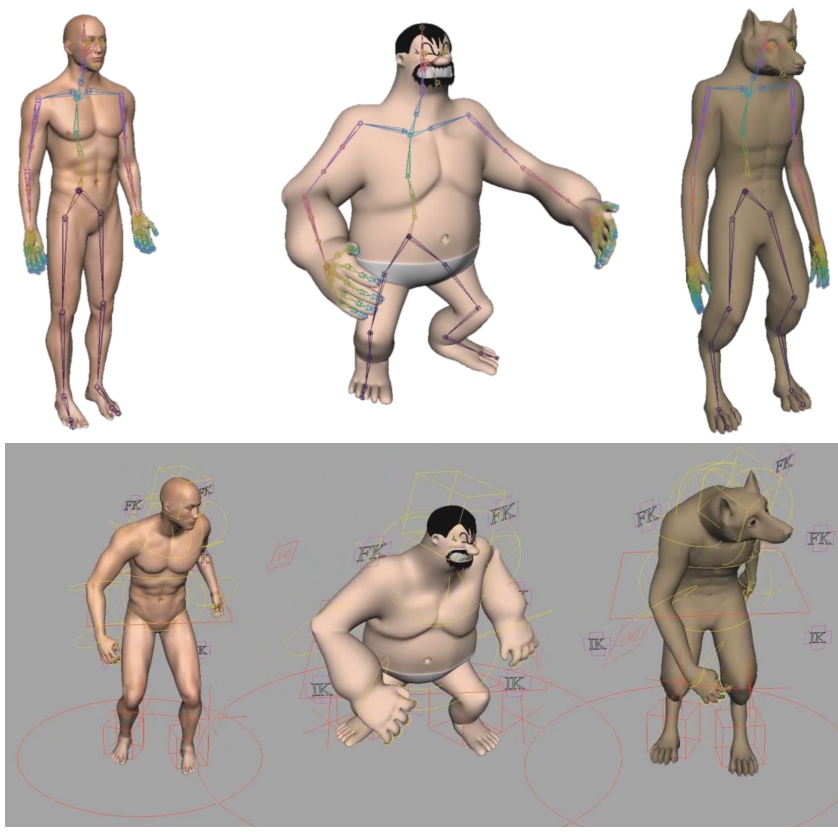

Figure 11: Top: Transfer of an articulated system. Bottom: Transfer of animation.

Proceedings of the Symposium on Geometry Processing, SGP '08, 1421-1430.

Lipman, Y., AND Funkhouser, T. 2009. Möbius voting for surface correspondence. ACM Trans. Graph., Proc. SIGGRAPH 28,3 .

M. Gilroy, Brian R. MacPherson, L. M. R. 2008. Atlas of anatomy. Thieme.

Mateus, D., Horaud, R., Knossow, D., Cuzzolin, F., And BOYER, E. 2008. Articulated Shape Matching Using Laplacian Eigenfunctions and Unsupervised Point Registration. In IEEE Conference on Computer Vision and Pattern Recognition (CVPR '08), IEEE Computer Society, 1-8.

MAYA-MUSCLE, 2013. http://images.autodesk.com/adsk/files/muscle.pdf.

Moore, K. L., AND Dalley, A. F. 1999. Anatomy Clinically Oriented, fourth ed. Lippincott Williams \& Wilkins.

Müller, M., Heidelberger, B., Teschner, M., AND Gross, M. 2005. Meshless deformations based on shape matching. ACM Trans. Graph. (Proc. of SIGGRAPH), 471-478.

Ovsjanikov, M., Ben-Chen, M., Solomon, J., Butscher, A., AND Guibas, L. 2012. Functional maps: a flexible representation of maps between shapes. ACM Trans. Graph. 31, 4, 30:1-30:11.

Patterson, T., Mitchell, N., AND Sifakis, E. 2012. Simulation of complex nonlinear elastic bodies using lattice deformers. ACM Trans. Graph. 31, 6 (Nov.), 197:1-197:10.

Pollard, N. S., ANd Zordan, V. B. 2005. Physically based grasping control from example. In Proceedings of the 2005 ACM SIGGRAPH/Eurographics symposium on Computer animation, ACM, New York, NY, USA, SCA ’05, 311-318.

Press, Teukolski, Vetterling, And Flannery. 2002. $\mathrm{Nu}$ merical Recipes in $\mathrm{C}++$. Cambridge University Press. 


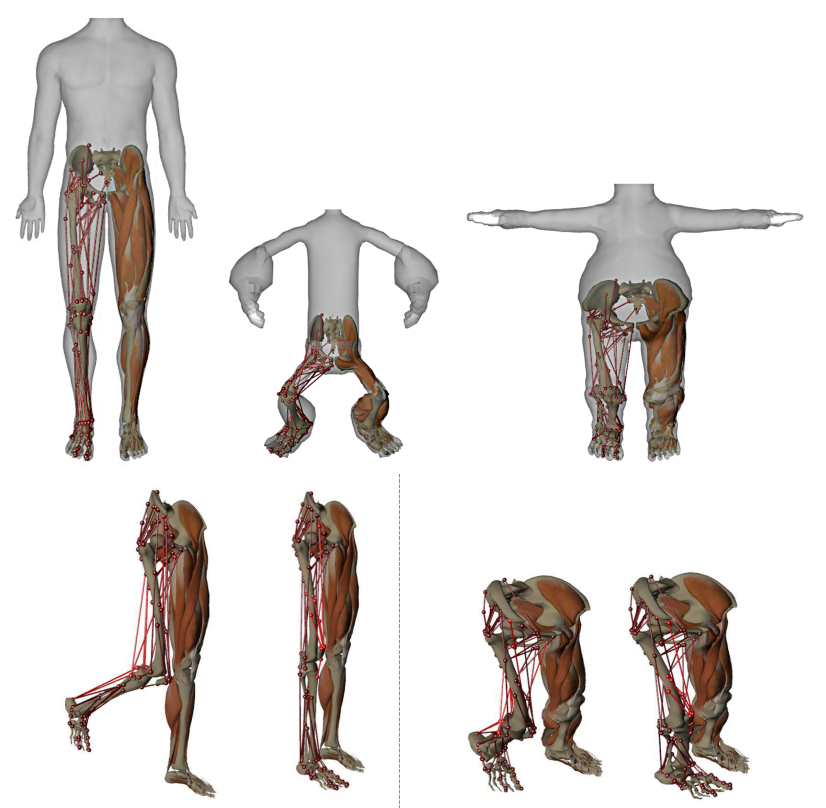

Figure 12: Top: Transfer of muscle lines of action. Bottom: knee ${ }^{585}$ movement using muscle control on both the source (zygote) and the ${ }_{587}$ target.

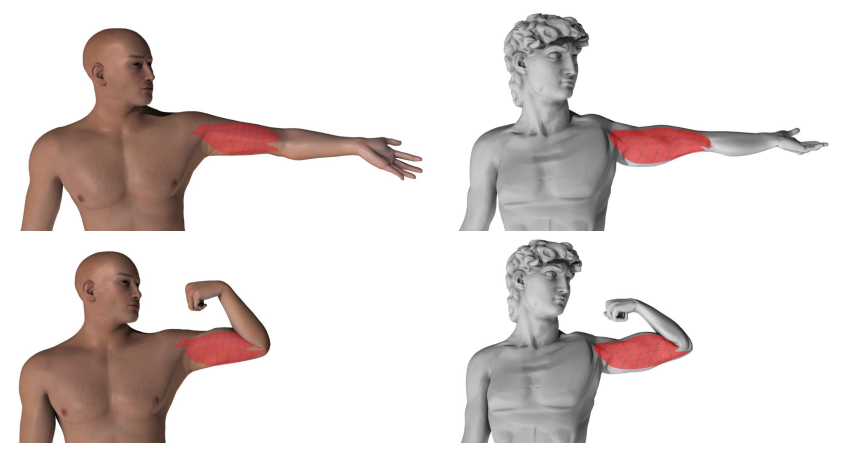

Figure 13: Top: Transfer of muscle and skin animation by using transferred muscles.
Scheepers, F., Parent, R. E., Carlson, W. E., and May, S. F. 1997. Anatomy-based modeling of the human musculature. In Proceedings of the 24th annual conference on Computer graphics and interactive techniques, ACM Press/AddisonWesley Publishing Co., New York, NY, USA, SIGGRAPH '97, 163-172.

Sifakis, E., Neverov, I., And Fedkiw, R. 2005. Automatic determination of facial muscle activations from sparse motion capture marker data. ACM Trans. Graph. 24, 3.

Sueda, S., Kaufman, A., And Pai, D. 2008. Musculotendon simulation for hand animation. ACM Transactions on Graphics 27, 3, 83:1-83:8.

Sumner, R. W., ANd Popović, J. 2004. Deformation transfer for triangle meshes. ACM Trans. Graph. 23, 3, 399-405.

Thelen, D. 2003. Adjustment of muscle mechanics model parameters to simulate dynamic contractions in older adults. ASME

\section{$125,1,70-77$.}

Wang, J. M., Hamner, S. R., Delp, S. L., and Koltun, V. 2012. Optimizing locomotion controllers using biologicallybased actuators and objectives. ACM Trans. Graph. 31, 4 (July), 25:1-25:11.

WATERS, K. 1987. A muscle model for animation threedimensional facial expression. SIGGRAPH Comput. Graph. 21, 4 (Aug.), 17-24.

WeI, Q., SuEdA, S., AND PAI, D. K. 2010. Biomechanical simulation of human eye movement. In Proceedings of the 5th international conference on Biomedical Simulation, Springer-Verlag, Berlin, Heidelberg, ISBMS'10, 108-118.

Wilhelms, J., AND VAN Gelder, A. 1997. Anatomically based modeling. In Proceedings of the 24th annual conference on Computer graphics and interactive techniques, ACM Press/Addison-Wesley Publishing Co., New York, NY, USA, SIGGRAPH '97, 173-180.

Zhu, Y., Sifakis, E., Teran, J., And Brandt, A. 2010. An efficient multigrid method for the simulation of high-resolution elastic solids. ACM Transcations on Graphics (Presented at SIGGRAPH 2010) 29, 2, 16:1-16:18.

Zordan, V. B., Majkowska, A., ChiU, B., And Fast, M. 2005. Dynamic response for motion capture animation. ACM Trans. Graph. 24, 3 (July), 697-701. 